\title{
EL ORDEN DEL ADJETIVO EN LA LENGUA DE SEÑAS ARGENTINA. UN ESTUDIO PRELIMINAR
}

\section{ADJECTIVE PLACEMENT IN ARGENTINE SIGN LANGUAGE. A PRELIMINARY STUDY}

\section{ROCÍO ANABEL MARTÍNEZ}

Consejo Nacional de Investigaciones Científicas y Técnicas (Conicet) y

Facultad de Filosofía y Letras de la Universidad de Buenos Aires

rociomartinez@conicet.gov.ar

\section{MARIANA MORÓN USANDIVARAS}

Facultad de Filosofía y Letras de la Universidad de Buenos Aires

marianamoron@filo.uba.ar

\section{RESUMEN}

En este artículo se presenta un estudio preliminar de los esquemas adjetivos en la Lengua de Señas Argentina (LSA) desde el Enfoque Cognitivo Prototípico (Lakoff, 1987; Langacker, 1987, 1991 y 2008). Para ello, se analizan cualitativa y cuantitativamente los esquemas adjetivos relevados en un corpus compuesto por 30 videograbaciones de señantes naturales de la LSA en relación con dos variables: (a) posición del adjetivo en la instanciación del nominal y (b) grado de contraste. Se busca definir la frecuencia de uso de las posiciones prenominal [Adj $\mathrm{N}$ ], posnominal [N Adj] e intranominal [N-Adj] del adjetivo respecto del núcleo sustantivo con el fin de encontrar cuál es la posición de preferencia de los señantes y la especificación en el uso de cada una de estas construcciones. En esta investigación se arriba a la conclusión de que en posición pospuesta, la preferida por los señantes, la construcción nominal conceptualiza una diferencia con alto grado de contraste con otras entidades similares presentes o latentes en el discurso mientras que en la posición antepuesta la diferencia que conceptualiza el adjetivo manifiesta un grado bajo de contraste. En cuanto a la posición intranominal, parece que no establece una relación de gradación de contraste como en los casos anteriores, sino que responde a la posibilidad que ofrece la LSA de incorporar morfológicamente ciertos atributos a sustantivos y estaría motivada por el principio de economía del signo (De Jonge, 2000).

Palabras clave: Lengua de Señas Argentina (LSA), Enfoque Cognitivo Prototípico, posición del adjetivo, grado de contraste. 


\section{ABSTRACT}

In this article we make a preliminary contribution to adjective schemas in Argentine Sign Language (LSA) within a Cognitive-Prototype Perspective (Lakoff, 1987; Langacker, 1987,1991 y 2008). We analyze qualitative and quantitatively adjective schemas that belong to real LSA signers' productions. This data, consisting in 30 videos, is analyzed with regards to two variables: (a) adjective placement within the nominal; (b) degree of contrast. We define the frequency of use of adjectives within the nominal schema, where prenominal [Adj $\mathrm{N}$ ], postnominal [N Adj] and intranominal [N-Adj] position can be found, in order to gain insight into the signer's preference regarding placement, as well as to better understand the specificity of each construction. In this paper, we conclude that postnominal position, which is the most frequently used by the signers of our data, the nominal constructions conceptualize a difference with a high degree of contrast amongst other similar entities that are explicit or implicit in discourse, while in prenominal position the difference that conceptualizes the adjective shows a low degree of contrast. Regarding intranominal position, it seems that there is no gradation of contrast, as in the former cases, but a different phenomenon involved: the possibility of the language to incorporate morphologically certain types of attributes within nouns may be motivated by the principle of economy of the sign (De Jonge, 2000).

Keywords: Argentine Sign Language (LSA), Cognitive-Prototype Perspective, Adjective placement, degree of contrast.

Recibido: 20.01.2016. Aceptado: 14.03.2016.

\section{INTRODUCCIÓN}

La Lengua de Señas Argentina (LSA, en adelante) ${ }^{1}$ es la lengua natural de las personas Sordas pertenecientes a la comunidad Sorda argentina ${ }^{2}$. Las personas Sordas han desarrollado en nuestro país un alto nivel de interacción Sordo/ Sordo, cues-

${ }^{1}$ Por convención en países hispanohablantes, las lenguas no orales se denominan utilizando en mayúsculas, primero, su modalidad de transmisión (Lengua de Señas para los países americanos hispanohablantes; Lengua de Signos para la península española) y, segundo, el lugar donde se encuentra la comunidad Sorda en cuestión (Argentina, Uruguaya, Chilena, Mexicana, etcétera). Si bien generalmente el lugar de procedencia coincide con el territorio total del país, hay casos donde existe más de una lengua de señas en un mismo país (y, consecuentemente, más de una comunidad Sorda). Tal es el caso de la Lengua de Signos Española y la Lengua de Signos Catalana o de la Lengua de Señas Brasilera (LIBRAS) y la Lengua de Señas Urubú-Kaapor (Ferreira Brito, 1984), entre muchos otros.

${ }^{2}$ La palabra Sordo se escribe con mayúsculas para referirse a la identidad lingüística y cultural de las personas que pertenecen a la comunidad Sorda (concepción socio-antropológica) y diferenciarse de la sordera como una condición audiológica (concepción clínica) (Padden, 1983 [1980]: 90; Wilcox, 2007: 1113). 
tión que les ha llevado no sólo a crear una lengua completa, compleja y distinta del español (Massone, 1993; Massone y Machado, 1994), sino también una cultura eminentemente visual (Massone, Simón y Druetta, 2003). Esta cultura propia de las personas Sordas no puede definirse simplemente como una serie de conductas diferenciales respecto de la mayoría oyente, sino que se refiere a un modo de estructurar simbólicamente el mundo que los rodea, como así también a sí mismos, que depende de la modalidad visual.

El objetivo general de este trabajo es aportar nuevos elementos descriptivos para la caracterización del esquema adjetivo en la LSA desde el Enfoque Cognitivo-Prototípico (Lakoff, 1987; Langacker, 1987, 1991 y 2008). Puntualmente, se pretende realizar un primer acercamiento al estudio de la posición de los posibles esquemas adjetivos (actualmente denominados verbos de estado) que se instancian en el nominal a partir de un corpus real de señantes naturales de la LSA. Se intentará definir con qué frecuencia se utilizan las posiciones (prenominal, posnominal e intranominal) de la unidad de análisis en el nominal. Esta información dará la pauta de cuál es la posición de preferencia de los señantes de la LSA, como así también algunas posibles especializaciones en el uso de las distintas construcciones. En la presente investigación se postula la hipótesis de que en la construcción [N Adj] se conceptualiza una diferenciación con otras entidades con un alto grado de contraste, mientras que en la construcción [Adj $\mathrm{N}$ ] hay una diferenciación con bajo o nulo contraste. La posición intranominal [N-Adj] parece no responder a esta gradación a nivel sintáctico entre mayor o menor contraste sino a la posibilidad que ofrece la LSA de incorporar morfológicamente ciertos atributos a sustantivos, lo que responde al principio de economía al que tiende toda lengua (De Jonge, 2000).

Para ello, se utiliza un cuerpo de datos que consiste en 30 videograbaciones de 24 personas Sordas señantes de la LSA. En todas las grabaciones ${ }^{3}$ se privilegia la construcción de discursos en contextos reales de producción, es decir, no se solicita a los señantes la elicitación de señas o frases sueltas ni descontextualizadas. Esta decisión metodológica surge de un principio teórico que considera que las unidades simbólicas -como es el caso del adjetivo- no son categorías a priori sino emergentes del discurso (Cf. Hopper y Thompson, 1984; Hopper, 1988).

\section{EL ADJETIVO}

En el presente trabajo se analizan esquemas adjetivos que se encuentran dentro de un nominal cuyo núcleo es un sustantivo. El adjetivo como clase de palabra modifica o predica de un sustantivo aportando diversos significados, es decir, el

\footnotetext{
${ }^{3}$ Siempre que se aluda a grabación en el artículo, se refiere a una realizada en video.
} 
adjetivo agrega información o rasgos que contribuyen a caracterizar la entidad denotada por el sustantivo (Real Academia Española, 2009: \$13). Consideramos que el fenómeno de la adjetivación se debe a "la experiencia humana básica de que un objeto puede tener una cualidad (o estar en un estado), de modo que se obstaculiza o impulsa determinado acto/acción relacionado con el soporte del atributo. El adjetivo significa una limitación o ensanchamiento del radio de la acción" (Fogsgaard, 1999: 588).

\subsection{La posición del adjetivo en el español ${ }^{4}$}

Existen varios estudios de gramática del español que abordan la cuestión de la posición sustantivo-adjetivo. En estos se dan al menos dos grandes tipos de explicación respecto de la posibilidad del español de utilizar adjetivos en posición antepuesta o pospuesta al sustantivo. Por un lado, desde perspectivas formales se sostiene que la posición del adjetivo se relaciona con las distintas subcategorizaciones que se le atribuyen (especificativos y explicativos; relacionales, calificativos y adverbiales) (Bosque, 1991; Demonte, 1999). Estas subcategorizaciones poseen propiedades que funcionan como restricciones en la colocación de los distintos adjetivos en el nominal. Por ejemplo, los adjetivos relacionales (costumbre mexicana) siempre tienen posición posnominal. Sin embargo, algunos de estos adjetivos pueden recategorizarse como calificativos $y$, cuando esto ocurre, pueden aparecer en posición prenominal (mexicana costumbre), aceptar adverbios de grado (muy mexicana costumbre) o el sufijo -ísimo (mexicanísima costumbre).

Por otro lado, estudios realizados desde perspectivas funcionales niegan que la subcategorización del adjetivo determine su posición en relación con el núcleo sustantivo. Desde este punto de vista, es la posición del adjetivo en relación con su núcleo en un contexto nominal la que posee un significado invariante: la diferenciación (Bolinger, 1954; Klein-Andreu, 1983). Este significado invariable posee dos categorías: diferente de otros/ diferente de si mismo (Diver, 1995; Huffman, 2001), diferente sin contrastel diferente con contraste (Klein-Andreu, 1983), o diferencia absolutal diferencia relativa (Martínez, 2006). Entonces, la anteposición del adjetivo a su núcleo [Adj N] se relaciona con la ausencia de contraste mientras la posposición con la presencia de contraste. Por ejemplo, en la silla blanca el adjetivo permite diferenciar la silla que se está conceptualizando en el discurso

\footnotetext{
${ }^{4}$ Si bien no es un estudio comparativo entre las lenguas de señas y lenguas orales, se presenta un breve panorama de los estudios sobre la posición del adjetivo en español porque es la lengua oral con la que está en contacto la LSA. Se pretende abordar estudios de gramáticas comparadas entre la LSA y el español en etapas posteriores, puesto que resulta fundamental para la formación profesional de carreras que involucran el conocimiento de ambas lenguas (intérprete LSA/E, docente de escuelas interculturales-bilingües LSA/E, docente de enseñanza de la LSA, etcétera).
} 
de otras posibles conceptualizaciones de sillas en la situación comunicativa dada. Mientras que en blanca nieve el adjetivo no contribuye a establecer una diferencia entre distintas conceptualizaciones de nieve en ese discurso.

Desde el ECP, se entiende la 'diferenciación' como una actividad de percepción y el 'contraste' como una relación particular que el hablante percibe entre dos o más posibles designados y el oyente reconstruye. Por ejemplo, en el siguiente fragmento de "El hombrecito del azulejo" de Mujica Láinez se encuentran dos adjetivos frágil y nuevos:

Martinito piensa que el niño, su amigo, va a morir, y le late el frágil corazón de cerámica. Ya nadie acudirá cantando a su escondite del zaguán; nadie le traerá los juguetes nuevos, para mostrárselos y que conversen con él. Quedará solo una vez más, mucho más solo ahora que sabe lo que es la ternura.

El adjetivo nuevos en posición pospuesta presenta una diferenciación por contraste. La conceptualización de juguetes nuevos distingue esos juguetes de otros posibles juguetes que se encuentran latentes en este discurso como los juguetes viejos del niño, precisamente, por la relación que puede establecerse en la oposición nuevo/viejo. Mientras que la posición antepuesta de frágil manifiesta diferencia sin contraste puesto que el designado del sustantivo (corazón) está suficientemente distinguido y no es necesario establecer una diferenciación con otros corazones.

\subsection{El adjetivo en las lenguas de señas y en la LSA}

El adjetivo como clase de palabras es una categoría problemática para los estudiosos de las distintas lenguas de señas en el mundo. Por ejemplo, en la Lengua de Señas Americana (ASL), que es la lengua de señas con mayor cantidad de estudios en el mundo, existen dos grandes posturas: por un lado, se lo toma como un subtipo de verbo (Klima y Bellugi, 1979), mientras que, por otro lado, se lo considera como un adjetivo (Padden, 1988; MacLaughlin, 1997; Wilbur, Malaia y Shay, 2012). Quienes pertenecen al primer grupo se detienen en mayor medida en la capacidad de este grupo léxico de atribuir propiedades a nominales ya formados, pero no analizan la participación de este grupo léxico en la construcción del nominal. Quienes pertenecen al segundo grupo, por el contrario, se centran tanto en los adjetivos que forman parte del nominal como en los que se encuentran en el predicado.

Un antecedente directo del presente estudio es el análisis del sintagma determinante de MacLaughlin (1997) desde el programa minimalista. En este trabajo, MacLaughlin (1997) observa que dentro del sintagma nominal el adjetivo en la ASL puede ocupar la posición prenominal o posnominal. Entre ambas posiciones 
se presentan las siguientes diferencias: (1) mientras los adjetivos prenominales tienen un orden más rígido, los posnominales tienen un orden más libre; (2) algunos adjetivos sólo aparecen en posición prenominal; (3) los adjetivos tienen diferencias en el significado dependiendo de la posición: los posnominales reciben una lectura predicativa; los prenominales una lectura atributiva; (4) los adjetivos prenominales y los posnominales son estructuralmente distintos.

Respecto del análisis de clases de palabras de la LSA, existe una gran cantidad de descripciones de las dos clases mayores, esto es, el sustantivo y el verbo (Curiel y Massone, 1993; Massone y Machado, 1994; Massone et al., 2000; Cvejanov, 2002; Massone y Martínez, 2012). Por el contrario, al igual que los estudios para otras lenguas de señas, la clase de los adjetivos ha resultado desde el inicio de la investigación lingüística de la LSA una cuestión problemática. Si bien hasta el momento no existen estudios que argumenten respecto de su inexistencia, lo que podría categorizarse como adjetivo se encuentra en la literatura como una subcategoría verbal, denominada verbo de estado. Puntualmente, Curiel y Massone (1993) consideran que el verbo posee doble función: una, principal, la predicación; otra, secundaria, la atribución. Aunque también pueden ser utilizados predicativamente, los verbos de estado son los encargados de cumplir con la función atributiva de manera exclusiva (Curiel y Massone, 1993: 31). De acuerdo con esta postura, los verbos de estado pueden tanto predicar de manera directa, es decir, sin la intervención de un verbo cópula, como cumplir una función atributiva ${ }^{5}$. Ahora bien, más allá de la función que cumplan, siempre se glosan junto a una partícula copulativa: SER-AZUL, ESTAR-PREOCUPADO.

Estos estudios fueron pioneros en la primera descripción científica de la LSA, utilizando principios de la lingüística. Dado que no había ningún estudio previo, el equipo de investigación dirigido por la Dra. Massone optó preferentemente por abordajes formales en la descripción de la gramática de la LSA. Si bien este equipo desde sus inicios realizó trabajo etnográfico en el seno de la comunidad Sorda argentina, la metodología propia de los abordajes formales no se caracteriza por realizar análisis basados en discurso sino en la oración. Consecuentemente, las clases de palabras han sido analizadas a partir de frases, esto es, haciendo foco en lo oracional más que en lo discursivo.

En una etapa posterior a estos estudios pioneros, consideramos que la única forma de comprender si existe una forma de conceptualizar esquemas adjetivos en la LSA, esto es, relaciones atemporales cuyo único participante focal es una

\footnotetext{
${ }^{5}$ Sostienen Massone y Machado (1994: 101): "Dada la inexistencia de verbo cópula en la LSA y en otras lenguas de señas (...), la predicación se realiza en forma directa a través de los verbos de estado, sin necesidad de lexicalizar la relación vacía de significación que manifiestan los verbos cópula en muchas lenguas orales".
} 
cosa (un esquema de sustantivo), es realizando un abordaje de lengua en uso. El adjetivo resulta una categoría más difícil de distinguir porque (dependiendo de la lengua) comparte propiedades con el sustantivo, con el verbo o con ambas. A causa de esta particularidad, en la tradición de análisis lingüístico de lenguas con mayor descripción, los adjetivos no siempre han sido considerados como una clase de palabras distinguible ${ }^{6}$. Sin embargo, esta capacidad del adjetivo de compartir propiedades con otras clases de palabras en otras lenguas del mundo no impide su identificación, siempre y cuando se entienda que entre las clases de palabras existen límites difusos, que sólo pueden observarse en el discurso (Langacker, 1987; 1991; Lakoff, 1987).

Hasta el momento, existen estudios de la LSA desde una perspectiva cognitiva que abordan el análisis de distintos mecanismos cognitivos, como así también ciertas expresiones lingüísticas. Por un lado, los mecanismos cognitivos analizados son las metáforas conceptuales, la metonimia conceptual y la iconicidad cognitiva (Martínez, 2012; Massone y Martínez, 2013; Martínez y Morón Usandivaras, 2013a y 2013 b). Por otro lado, el análisis de expresiones lingüísticas se ha centrado específicamente en la problematización del funcionamiento de los verbos de estado dentro del sistema de clases de palabras de la LSA (Martínez, 2013a y 2015). Bajo esta misma problemática, existen estudios sobre la forma de conceptualizar la dimensión en esquemas nominales concretos de la LSA (Martínez, 2013b y 2014a). Estos estudios permitieron identificar dos grupos nominales: por un lado, nominales donde los sustantivos modifican su estructura morfológica para agregar un significado relativo a la dimensión (EDIFICIO-ALTO, NARIZ-LARGA) y, por otro lado, nominales donde se recurre a una construcción sintáctica (BARCO GRANDE, VENTANA CUADRADA). Se observa que la conceptualización de la dimensión en esquemas nominales concretos de la LSA se instancia sintáctica o morfológicamente de acuerdo con dos operaciones cognitivas: el tipo de proceso metonímico de creación léxica del sustantivo y dos propiedades fonológicas del sustantivo (contacto y bi/unimanualidad). Asimismo, Martínez (2014b) ha realizado un primer abordaje semántico de tres adjetivos que indican temporalidad en la LSA, a saber: VIEJO, ANTIGUO y ANTERIOR. En ese estudio se observa que los mencionados adjetivos recurren a un esquema escalar que, en ocasiones, presenta una norma como región en el dominio conceptual.

La presente investigación pretende avanzar en el estudio de los posibles adjetivos en la LSA en un contexto nominal. Hasta el momento, no hay estudios que aborden el tema de la posición de los posibles adjetivos en el nominal.

\footnotetext{
${ }^{6}$ De hecho, en las primeras descripciones lingüísticas de lenguas como el latín o el griego no había una distinción entre el adjetivo y el sustantivo.
} 


\section{ENFOQUE COGNITIVO PROTOTÍPICO}

El Enfoque Cognitivo Prototípico (ECP) concibe que la gramática emerge del discurso (Hopper: 1988); la gramática es emergente en el sentido de que su estructura y sus regularidades surgen del discurso y se establecen en un proceso continuo en cada acto de comunicación. En otras palabras, la gramática es entendida como series de recurrencias que vienen de diferentes géneros y de múltiples tipos discursivos y situaciones comunicativas. Esas recurrencias se reconocen como gramaticales cuando un número suficiente de ellas puede ser identificado como parte de un subsistema. Estas rutinas son utilizadas y reutilizadas por los hablantes/señantes porque son percibidas como útiles y exitosas. Desde esta perspectiva, se impone un enfoque basado en el uso; se concibe el signo como una unidad motivada por la función comunicativa. La sintaxis, como parte de la gramática, está motivada por la intención comunicativa y responde al contenido semántico de lo que se quiere lograr (Diver, 1995: \$1.4; Contini-Morava, 1995: \$3.3; Geeraerts, 2006; Huffman, 2001: \$1; Langacker, 2008: \$2 y García, 2009: \$1.2.2).

Para Langacker (1987 y 2008), las estructuras semánticas son conceptualizaciones confeccionadas por las especificaciones de las convenciones lingüísticas, por lo tanto, el análisis sintáctico-semántico requiere una caracterización explícita de la estructura conceptual. Una experiencia mental es la ocurrencia de un evento cognitivo. Una experiencia sensorial primaria es un evento cognitivo evocado directamente por el estímulo de un órgano sensorial (el ojo ve una casa). La correspondiente imagen sensorial no es otra cosa que la ocurrencia de un evento cognitivo equivalente en ausencia del estímulo externo (el ser humano puede re-crear esa imagen de la casa cuando esta no se encuentra delante de sus ojos). La relación entre el hablante/señante (y su interlocutor) y la situación que está conceptualizando implican la construcción de imágenes.

Desde el ECP, un esquema lingüístico es una abstracción conceptual creada a partir de una serie de instanciaciones que han resultado exitosas al hablante/ señante, puesto que han logrado satisfacer sus necesidades comunicativas. Esta abstracción sólo puede ser abordada en instanciaciones discursivas concretas. Las unidades lingüísticas "occur in particular settings, from which they derive much of their import, and are recognized by speakers as distinct entities only through a process of abstraction" (Langacker, 1987: 401).

El ECP sostiene, además, que el hablante/señante busca transparencia en su mensaje, es decir, que el mensaje logre el objetivo comunicativo de la manera más efectiva. Por lo tanto, el hablante/señante busca la correlación una forma/un significado por claridad. En consecuencia, se presupone que todas las formas son significativas, es decir, cada adjetivo tiene un significado específico en los distintos contextos de uso en los que suele aparecer. Por consiguiente, cada forma y cada construcción sintáctica deben ser estudiadas en relación con su significado en el 
discurso y en la situación comunicativa en la que son producidas teniendo en cuenta criterios cualitativos y cuantitativos ya que la frecuencia de uso está en la base de la gramaticalización de las formas y las construcciones.

\section{METODOLOGÍA}

Acorde a los principios del ECP expuestos más arriba, la intención de realizar una descripción que dé cuenta del uso de las formas en sus verdaderos contextos de aparición implican necesariamente una metodología no solamente cualitativa sino también cuantitativa y un trabajo sobre corpus auténtico. A continuación, en 4.1 se realiza una descripción del cuerpo de datos, mientras que en 4.2. se entregan precisiones respecto de la metodología de análisis cualitativa y cuantitativa.

\subsection{Cuerpo de datos}

El cuerpo de datos del presente artículo se encuentra compuesto por 30 videograbaciones de 24 personas Sordas adultas que forman parte de la comunidad Sorda argentina. Todos ellos poseen la Lengua de Señas Argentina (LSA) como su lengua natural y primera, y la utilizan con alta frecuencia en intercambios cotidianos con miembros de la comunidad Sorda de nuestro país.

Si bien en todas las grabaciones se privilegió la construcción de discursos en contextos reales de producción -esto es, nunca se solicitó la elicitación de señas sueltas ni descontextualizadas-, de acuerdo con la temática y con algunas características diferenciales, estas grabaciones se dividieron en seis grupos:

(i) El tiempo (CIIi). Este grupo de grabaciones tomó como punto de partida una temática: el tiempo. Se les pidió a cuatro (4) personas Sordas que observaran un libro de fotografías denominado ¿Qué es el tiempo? En este libro, cada una de las imágenes da cuenta de distintas formas de concebir el tiempo en la cultura occidental. Luego, se les pidió que seleccionaran dos o tres fotografías y que contaran en LSA lo que habían visto en ellas, mientras eran grabados. Una persona Sorda fue la encargada de dar las indicaciones en LSA. Total de grabaciones: 4 .

(ii) Historias de vida (CIIii). Se solicitó a dos (2) personas Sordas que narraran sus historias de vida en LSA. Los encargados de solicitar estas narrativas fueron personas muy allegadas a ellos, de manera tal de generar un clima de confianza apropiado para la narración de hechos pasados que los involucran como protagonistas. Total de grabaciones: 2 . 
(iii) Relatos de accidentes (CIIiii). Se solicitó a seis (6) personas Sordas que narraran en LSA una situación límite en la que se hayan visto involucrados. Una persona Sorda que colabora con la investigación fue la encargada de dar la temática a desarrollar como así también de realizar la grabación. Total de grabaciones: 6 .

(iv) Conversaciones (CIIiv). Se solicitó a diez (10) personas Sordas que eligieran un tema de conversación a elección (por ejemplo, la familia, un viaje, organización de actividades, etcétera). A continuación, debían conversar en parejas sobre el tema elegido, mientras eran grabados. Total de grabaciones: 7.

(v) Entidades no animadas (lugares y objetos): descripción y narración (CIIv). Se solicitó a cuatro (4) personas Sordas que eligieran una entidad no animada, especialmente un lugar o un objeto, que resulte de su interés y que contaran todo lo que supieran de ella (es decir, describir y narrar). La encargada de solicitar y grabar estos datos fue la primera autora del presente trabajo; las personas Sordas grabadas son personas con las que tiene un alto grado de interacción. Total de grabaciones: 6 .

(vi) Entidades animadas humanas: descripción y narración (CIIvi). Se solicitó a tres (3) personas Sordas que eligieran una persona muy conocida (por ejemplo, un actor, un personaje histórico, un deportista, un músico). Luego, debían contar de la manera más detallada posible todo lo que supieran de esta persona: descripción física, hechos importantes en su vida, etcétera. Al igual que en (v), la encargada de solicitar y grabar estos datos fue la primera autora del presente trabajo; las personas Sordas grabadas son personas con las que tiene un alto grado de interacción. Total de grabaciones: 5 .

Debido a que algunos señantes participaron en más de una grabación, la cantidad de personas Sordas contabilizada por tipo de muestra difiere de la cantidad total de personas Sordas, esto es, son 24 personas en total. Se contabiliza un total de 30 videos y una duración total de dos horas con cinco minutos (02:05:00) ${ }^{7}$.

Todas las grabaciones fueron transcriptas en glosas, de acuerdo con las convenciones utilizadas en el área de la lingüística de la lengua de señas (Massone y Machado, 1994: 92-95). Las glosas intentan representar, de la manera más precisa y detallada posible, la gramática de la lengua de señas bajo estudio, utilizando una determinada lengua escrita. En este caso en particular, se glosaron en español

${ }^{7}$ Cabe aclarar que estas grabaciones forman parte de un cuerpo de datos mayor, correspondiente a la tesis doctoral de la primera autora de este trabajo, quien desde 2010 se encuentra realizando investigación etnográfica con miembros de la comunidad Sorda argentina. 
escrito todos los discursos grabados en la LSA. Cada seña se escribe en mayúsculas en el orden en el que fue producida por el señante (PASADO PRO1sg NIÑO). En el caso de que una única seña contenga más de un significado, las glosas en español dan cuenta de cada uno de ellos uniendo las palabras con guiones (LLORAR-DESCONSOLADAMENTE). Cuando la seña posee información gramatical relevante, ésta se escribe en minúsculas entre paréntesis, a continuación de la glosa en mayúsculas, por ejemplo, COMER(hab.) o LINDO(intens.). Asimismo, todos los rasgos no manuales con valor sintáctico o pragmático son glosados en una línea superior a la glosa manual, marcando su inicio y fin:

$$
\text { PRO1sg }{ }_{1} \text { CONTAR }_{3 \mathrm{pl}} \text { QUÉ }
$$

int./ CUad. ${ }^{8}$

Traducción: ¿Qué les voy a contar?

\subsection{Precisiones metodológicas sobre el análisis cualitativo y cuantitativo}

En la presente investigación se denominó Unidad de Análisis (UA) a aquellas señas de la LSA instanciadas en el cuerpo de datos como atributos en un contexto nominal (actualmente denominados verbos de estado en función atributiva, posibles adjetivos). En dicho cuerpo de datos se identificaron un total de 317 UA en contexto nominal.

En las UA identificadas se analizaron las variables cualitativas posición y grado de contraste. En ambas variables, el hecho de que cada UA se encuentre en un contexto discursivo particular permitió recabar información relevante respecto de los objetivos comunicativos del hablante y, en consecuencia, de las motivaciones que podrían explicar la manera particular de instanciarse de cada UA. Las variables posición y grado de contraste surgen del contraste entre literatura disponible hasta el momento respecto de la categoría adjetivo en otras lenguas y lo observado en un primer estudio exploratorio del cuerpo de datos.

En primer lugar, respecto de la posición de la UA en el nominal, se identificaron tres posiciones: (1) prenominal; (2) posnominal, e (3) intranominal. La posición prenominal [Adj $\mathrm{N}$ ] es aquella en la que el adjetivo se encuentra en primera posición, mientras el sustantivo ocupa un segundo lugar (GRANDE MONTAÑA). La posición posnominal [ $\mathrm{N}$ Adj] tiene un orden inverso, esto es, el sustantivo ocupa la primera posición y el adjetivo la segunda posición (CASA NUEVA). Por último, la posición intranominal [N-Adj] ya no es sintáctica sino morfológi$\mathrm{ca}$, puesto que una serie de sustantivos pueden -por motivaciones semánticas y fonológicas- incluir atributos en su morfología. Como se ha mencionado, Mar-

${ }^{8}$ Int: interrogación. CUad: cuerpo adelantado. 
tínez (2014a) analiza las posibles motivaciones que llevan a señas de dimensión (ALTO, GRANDE, etcétera) en nominales de la LSA a instanciarse en la morfología (EDIFICIO-ALTO) o en la sintaxis (BARCO GRANDE) y concluye que esta diferencia en la instanciación depende del tipo de proceso metonímico de creación léxica del sustantivo y dos propiedades fonológicas del sustantivo (contacto y bi/ unimanualidad).

En segundo lugar, el grado de contraste es una variable que se adaptó de los estudios de Bolinger (1954) y Klein-Andreu (1983). El primer autor considera que en los casos donde es posible la alternancia en el orden de palabras del español, el hablante reconoce una diferencia en el significado 9 . En este estudio, concluye que en todos los casos de alternancia en el orden de palabras del español se sigue el mismo patrón lingüístico, a saber: que en cada cláusula o frase hay una relación entre la posición de los elementos y el hecho de que sean presupuestos, no contrastivos, conocidos o atribuidos al contexto, o bien nuevos, contrastivos y desconocidos. A partir de la propuesta de Bolinger (1954), Klein-Andreu (1983) toma la variable presencialausencia de contraste para analizar el orden del adjetivo en el nominal. Ahora bien, puesto que la LSA constituye una lengua distinta del español, es necesario analizar las variables grado de contraste y posición en la LSA para saber si existe una asociación entre ambas, o si existen otras motivaciones que puedan justificar la alternancia en la posición del adjetivo respecto del sustantivo en la LSA.

En este artículo, consideramos que se observa el significado [contraste alto] si: (i) el adjetivo distingue entre otros posibles designados del sustantivo (el edificio angosto); (ii) informa la atributabilidad del adjetivo al sustantivo (aji picante); (iii) otorga un atributo al sustantivo (bailarín profesional); (iv) enfatiza la atributabilidad del sustantivo (payaso gracioso). Por el contrario, se observa el significado [contraste bajo o nulo] si: (i) el adjetivo no distingue con claridad entre otros posibles designados del sustantivo (acalorado debate); (ii) el adjetivo es suficientemente distinguido (aplastante mayoría); (iii) el adjetivo es presupuesto por el sustantivo (pequeña hormiga); (iv) el adjetivo está culturalmente atribuido al sustantivo (bendita Madre de Dios).

Entonces, por un lado, se realizará un primer abordaje cualitativo y cuantitativo de las distintas posiciones de la UA en el nominal, esto es, prenominal, posnominal e intranominal. Por otro, se propone analizar si existe una relación entre las mencionadas posiciones de las UA en el contexto nominal y el grado de contraste que establecen en el discurso. Para ello, se recurrirá a la estadística descriptiva. Esta ayudará a determinar la frecuencia relativa de uso de los distintos tipos de colocación de adjetivo en contexto nominal, lo que brindará un primer panorama de la

\footnotetext{
${ }^{9}$ Bolinger (1954) analiza el fenómeno del orden de palabras del español en los siguientes casos: sujeto-verbo, verbo-objeto, adjetivo-sustantivo, adverbio-verbo, coordinado-coordinado, superordinado-subordinado.
} 
utilización real de la UA por parte de los señantes naturales de la LSA. En adición, se cruzarán las dos variables cualitativas (posición y grado de contraste) para observar si entre ambas hay independencia o si existe una motivación semántica. En esta segunda parte del análisis se utilizará la Prueba de $X^{2}$, que es precisamente un test de independencia de variables cualitativas.

\section{RESULTADOS}

De las tres posiciones sintácticas de la UA que se detectaron en el cuerpo de datos (prenominal, posnominal e intranominal), en la Tabla I se observa una marcada preferencia por la posposición (78\%) [N Adj]. Las otras dos opciones posicionales tienen menor frecuencia relativa de uso: prenominal [Adj $\mathrm{N}$ ] posee un 13\%, mientras que intranominal [N-Adj] tiene un $10 \%$.

Tabla I. Posición de la UA.

\begin{tabular}{|l|l|l|}
\hline Posición & FA & FR $^{10}$ \\
\hline PreN & 40 & $13 \%$ \\
\hline PosN & 246 & $78 \%$ \\
\hline IntrN & 31 & $10 \%$ \\
\hline Total & 317 & $100 \%$ \\
\hline
\end{tabular}

Esta diferencia en la frecuencia relativa de uso de las tres construcciones da la pauta de que [N Adj] constituye una rutina altamente convencionalizada para los señantes de la LSA. Ahora bien, desde el ECP se supone que cuando más de una expresión lingüística (sea esta una unidad simple o compleja) desarrolla una misma función en el discurso, hay una tendencia a la especialización, de manera que a cada expresión lingüística le corresponden usos diferenciales. En este estudio preliminar se analizarán algunos casos para realizar una primera reflexión sobre el funcionamiento de cada construcción.

\section{(a) Viaje a Salta (narración/descripción) (CIIvA) \\ (i) LINDO CAMINO-SINUOSO IR-HACIA-ARRIBA \\ (ii) GRANDE}

${ }^{10}$ Las siglas FA hacen referencia a la frecuencia absoluta, es decir, la cantidad de veces que se repite un valor dentro de un parámetro (posición del esquema adjetivo, en este caso) y FR equivale a frecuencia relativa, o sea, un tanto por uno, sin embargo, hoy día es bastante frecuente hablar siempre en términos de tantos por ciento o porcentajes, por lo que esta medida resulta de multiplicar la frecuencia relativa por 100 . 
MONTAÑA(pl)

LLEGAR-LOCa LEJOS-ARRIBA MIRAR-HACIA-ABAJO CASA PAISAJE $[\ldots]^{11}$

(b) Organizar un día de pesca (conversación entre dos personas Sordas) (CIIivG)

A: PRO1 GRUPO PRO1 YA SENTARSE-EN-EL-AUTO(2m) COMPLETO IRSE MANEJAR++ LEJOS(intens) TOMAR-MATE CONVERSAR QUÉ PENSAR TEMA IMPORTANTE GUSTAR GRANDE MEDIANO CHICO NO-IMPORTAR++ [...]

S: BIEN(2M) PRO1 GUSTAR SOÑAR GUSTAR (i) GRANDE(intens) SU$\underline{\text { RUBÍ }}^{12}$

(c) Comprar una casa (narración/descripción) (CIIvB) CEfr,OJsc,LAab. _CEarr, OJab.

PRO1 QUERER BUSCAR COMPRAR (i) CASA NUEVA I _LAmord.

BUSCAR(cont) VER GUSTAR I CEfr. CEfr.LAest CEfr.LAab

ME-JOROBARON (ii) CASA ANTIGUA(intens) DESTRUIDA

DEBER PINTAR ARREGLAR+++ NO DEJAR I

BUSCAR(cont) QUERER (iii) CASA MENOS ANTIGUA I NUEVA ALGO BUSCAR(cont) ENCONTRAR(pret) (iv) CASA BUENA NORMAL PEQUE$\underline{\tilde{N}}{ }^{13}$

(d) Congreso de la Nación Argentina (narración/descripción) (CIIvD)

INGRESAR PUERTA (i) PUERTA-PESADA ALTA _LEaf.HOad. _LEaf.HOad.

INGRESAR (ii) BAÑO PEQUEÑO (iii)PUERTA(pl.) PEQUEÑA(pl.) BAÑO _LEaf.HOad._OJsc.LAest.HOad

HACER-PIS (iv) MINGITORIO PEQUEÑO-1 PEQUEÑO-2 DIFERENTE I SALIR (v) PASILLO-LARGO(rap.) GRANDE PASILLO-LARGO LEJOS-IX LEJOS-IX ${ }_{j}^{14}$

${ }^{11}$ Traducción al español: "Ascendíamos por un lindo camino sinuoso entre grandes montañas. Llegamos al punto superior y observamos todo lo que se encontraba abajo, casas, paisajes [...]".

${ }^{12}$ Traducción al español: "A: Nos subimos todos al auto y nos vamos muy lejos. En el viaje vamos a tomar mate, conversar, vamos a pensar sobre un tema importante: si nos gusta que [el pez] sea grande, mediano o pequeño -no importa- [...]. S: Muy bien. A mí me gustaría un gran surubí".

${ }^{13}$ Traducción al español: "Quería comprar una casa nueva. Busqué por un tiempo y vi una que me gustó, pero me jorobaron porque era una casa muy antigua y destruida. Había que pintarla y arreglarla así que la dejé. Continué buscando porque quería una casa menos antigua. Una nueva, algo así buscaba. Al final, encontré una casa buena, normal y pequeña".

${ }^{14}$ Traducción al español: "Al ingresar se observa una puerta pesada y alta y luego un baño muy 
El ejemplo (a) introduce una seña adjetiva en posición prenominal GRANDE que acompaña al sustantivo MONTAÑA(pl). En (b) se observa la misma seña de dimensión, pero intensificada (GRANDE(intens) SURUBÍ), también en posición prenominal [Adj N]. En (c) se encuentran cuatro UA en posición pospuesta al núcleo sustantivo casa [N Adj]: (i) NUEVA, (ii) ANTIGUA DESTRUIDA, (iii) MENOS ANTIGUA NUEVA y (iv) BUENA NORMAL PEQUEÑA y en (c) dos esquemas adjetivos en posición intranominal (PUERTA-PESADA y PASILLO-LARGO).

La seña adjetiva de (a) acompaña a un sustantivo en un contexto descriptivo GRANDE MONTAÑA(pl). El adjetivo contribuye a caracterizar la entidad designada por el sustantivo. El señante no está estableciendo una diferencia con otras entidades similares sino que simplemente describe y presenta 'las montañas'. La significación del adjetivo forma parte de la conceptualización del sustantivo; en nuestra cultura predomina el presupuesto de que las montañas son grandes, en otras palabras, el adjetivo está presupuesto por el sustantivo. Es un caso de contraste nulo.

En (b) la seña adjetiva modifica a un participante focal que se introduce por primera vez en el discurso (SURUBÍ). En este contexto, la seña GRANDE en posición prenominal contribuye a recortar la designación del núcleo sustantivo pero no parece generar un contraste significativo con otras entidades del mismo tipo, por lo tanto, puede decirse que la intención comunicativa del señante no es establecer una diferenciación entre designados similares presentes en el discurso. El grado de contraste que manifiesta el esquema adjetivo es muy bajo. En este caso, el contraste es bajo porque GRANDE(intens) SURUBÍ se encuentra en relación con lo expresado por el otro señante $A$ GRANDE MEDIANO CHICO, que conceptualiza cualquier pez de cualquier tamaño. La intención comunicativa del señante $S$ es expresar su deseo de pescar 'un surubí grande', por eso, especifica tanto al sustantivo (pez-surubí) como selecciona un adjetivo de entre los tres posibles según el señante $A$.

A diferencia de (a y b), la intención comunicativa del señante de (c) es claramente establecer una diferencia entre las conceptualizaciones del sustantivo casa. La primera designación (CASA NUEVA) designa una entidad que va a ser el tema de la narración. Cuando la seña CASA se instancia en los nominales (ii) y (iv) conceptualiza entidades específicas, y la señante se ve en la necesidad de diferenciar cada una de ellas de los otros designados del mismo tipo, o sea, diferenciar claramente la conceptualización del nominal (ii) de la conceptualización de (iv). Entonces, los atributos que acompañan al sustantivo CASA en (ii) y (iv) contribuyen a recortar la designación, generando un contraste con otras entidades

pequeño, con puertas muy pequeñas y un mingitorio muy pequeño. En contraste, saliendo hay un pasillo largo y grande que permite ver a lo lejos en ambas direcciones". 
mencionadas y presentes en ese discurso. El alto grado de contraste entre estos dos nominales se manifiesta claramente en la acumulación atributos que permite recortar la designación y se manifiesta sintácticamente en la coordinación de adjetivos en posición pospuesta.

Por último, en (d) la posición del adjetivo en el nominal no se manifiesta sintácticamente sino morfológicamente, dado que en una sola seña se encuentran el sustantivo y su adjetivo. La motivación de la posición intranominal de la UA no sería, como en los casos anteriores, la intención comunicativa del hablante de diferenciar con mayor o menor precisión la entidad designada de otras similares sino que estaría motivada por el principio de economía del signo lingüístico (De Jonge, 2000). En (d), las señas relativas al significado \{PUERTA\} y $\{$ PASILLO\} son modificadas morfológicamente para agregar un nuevo morfema atributivo con el significado $\{$ PESADO $\}$ y $\{$ LARGO $\}$, respectivamente. En ambos casos, los morfemas atributivos agregan significados relacionados con la propiedad física o la dimensión, que son dos dominios cognitivos cercanos al dominio básico del espacio. ${ }^{15}$ La modalidad viso-espacial de la LSA permite al señante expresar en una sola seña con total claridad y con el mínimo esfuerzo posible una conceptualización que, en lenguas orales como el español, implicaría el uso de dos o más palabras relacionadas sintácticamente.

Ahora bien, si se cruza la variable posición prenominal/posnominal, puesto que son los dos valores que se instancian sintácticamente, con la variable grado de contraste se observa que es posible establecer una asociación entre la posición prenominal y un grado de contraste bajo o nulo y la posición posnominal y un grado de contraste alto. La Tabla II muestra que en la posición prenominal prevalecen los casos que establecen bajo o nulo contraste ( $80 \%$ de la totalidad UA en posición prenominal), en tanto que en la posición posnominal el señante manifiesta una predilección por los casos que revelan un alto contraste $(93 \%$ de la totalidad de casos en posición posnominal).

Tabla II. Grado de contraste de la UA en posición prenominal y posnominal.

\begin{tabular}{|c|c|c|c|c|c|c|}
\hline Grado de contraste & \multicolumn{2}{|c|}{ Contraste bajo/nulo } & \multicolumn{2}{|c|}{ Contraste alto } & \multicolumn{2}{|c|}{ Total } \\
\hline \multirow[b]{2}{*}{ Prenominal } & \multicolumn{2}{|c|}{32} & \multicolumn{2}{|c|}{8} & \multicolumn{2}{|c|}{40} \\
\hline & $64 \%$ & $80 \%$ & $3 \%$ & $20 \%$ & $14 \%$ & $100 \%$ \\
\hline & \multicolumn{2}{|c|}{18} & \multicolumn{2}{|c|}{228} & \multicolumn{2}{|c|}{246} \\
\hline Posnominal & $36 \%$ & $7 \%$ & $97 \%$ & $93 \%$ & $86 \%$ & $100 \%$ \\
\hline
\end{tabular}

${ }^{15}$ Ver el estudio de la dimensión en esquemas nominales concretos de la LSA en Martínez (2014a). 


\begin{tabular}{|l|r|r|r|r|r|r|}
\hline \multirow{2}{*}{ Total } & \multicolumn{2}{|c|}{50} & \multicolumn{2}{c|}{236} & \multicolumn{2}{c|}{286} \\
\cline { 2 - 7 } & $100 \%$ & $17 \%$ & $100 \%$ & $83 \%$ & $100 \%$ & $100 \%$ \\
\hline
\end{tabular}

$X^{2}=121,004 ; \mathrm{gl}=1 ; p<0.05$

Valor de referencia $=3,84$

De acuerdo con los resultados de la Tabla II, dado que el valor calculado de $X^{2}$ (121) es mayor al del valor de referencia para grado de libertad 1 con una seguridad del $95 \%(\alpha=0,05)$, y dado que la probabilidad asociada al valor de $X^{2}$ calculado es menor a 0,05 (valor de $\alpha$ ), se puede confirmar la existencia de una relación entre las dos variables analizadas. Esto es, el grado de contraste tiene implicancias en la posición sintáctica que adopta la UA en la estructura del nominal.

En resumen, puede observarse en los esquemas adjetivos una gradación del nivel de contraste acorde a la posición que ocupa en relación con el núcleo sustantivo. En la posición antepuesta, si bien la diferenciación puede marcar un cierto contraste, como en el ejemplo (b), éste es menor que en los casos de posición pospuesta [N Adj] como en el caso de (c). La posición intranominal parece no responder a esta gradación entre mayor o menor contraste sino a la posibilidad que ofrece la lengua de señas de incorporar morfológicamente ciertos atributos a la misma seña, lo que responde al principio de economía al que tiende toda lengua.

\section{CONCLUSIONES}

Para concluir, puede observarse en el cuerpo de datos una preferencia de los señantes por la posición posnominal [ $\mathrm{N}$ Adj], puesto que hay una frecuencia relativa de uso muy alta (78\%). No obstante, la LSA permite otras opciones de instanciación de atributos en el nominal (posición prenominal, en la sintaxis, y posición intranominal, en la morfología del sustantivo) que, aunque posean una frecuencia relativa de uso más baja, resulta necesario explicar. Para encontrar primeras respuestas, se realizó un abordaje cualitativo y cuantitativo de las variables posición y grado de contraste, con el objetivo de observar si existe una conexión entre ambas. Los resultados de este análisis indican que, cuando no hay restricciones semánticas y fonológicas (como es el caso de los atributos que se instancian en posición intranominal), el orden relativo del adjetivo y del sustantivo en el nominal es significativo, es decir, es posible observar un significado posicional que favorece o desfavorece el contraste con otros posibles designados del mismo tipo.

En base a estos resultados, la mayor frecuencia relativa de uso de la posición 
posnominal respecto de las demás posiciones permite observar que la principal preocupación de los señantes del cuerpo de datos en la construcción de nominales es recortar entidades en el discurso de manera diferencial y contrastiva.

\section{REFERENCIAS}

Bolinger, Dwight. (1954). Meaningful word order in Spanish, Boletín de Filología, 7, 45-56.

Bosque, Ignacio. (1991). Las categorias gramaticales. Relaciones y diferencias. Madrid: Síntesis.

Contini-Morava, Ellen. (1995). Introduction: On linguistic sign theory. En: Contini-Morava, E. y B.S.Goldberg (Eds.). Meaning as Explanation: Advances in Linguistic Sign Theory. Berlin: W. de Gruyter, 1-39.

Curiel, Mónica y Massone, María I. (1993). Categorías gramaticales en la Lengua de Señas Argentina. Revista de Lingüistica Aplicada, 31. 27-53.

Cvejanov, Sandra (2002). Incorporación en verbos de movimiento de la lengua de señas argentina. Tesis de maestría. Universidad Nacional del Comahue.

De Jonge, Bob. (2000). Estudio analítico del signo lingüístico. Teoría y descripción, Foro Hispánico. Revista Hispánica de los Países Bajos, 17, pp. 7-14.

Demonte, Violeta. (1999). El adjetivo: clases y usos. La posición del adjetivo en el sistema nominal. En: Bosque, I. y Demonte, V. (Coords.). Gramática descriptiva de la lengua española. Madrid: Espasa. 129-216.

Diver, William. (1995). Theory. En: Contini-Morava, E. y B.S.Goldberg (Eds.). Meaning as Explanation: Advances in Linguistic Sign Theory. Berlin: W. de Gruyter, 43-114.

Ferreira Brito, Lucinda. (1984). Similarities and Differences in Two Brazilian Sign Languages. Sign Language Studies, 42, 45-56.

Fogsgaard, Lene. (1999). Las clases de palabras. Estudios de Lingüistica Cognitiva II. Alicante: Universidad de Alicante, 575-592.

García, Érica. (2009). The Motivated Syntax of Arbitrary Signs. Cognitive constraints on Spanish clitic clustering. Amsterdam / Philadelphia: John Benjamins.

Geeraerts, Dirk. (2006). Introduction: A rough guide to Cognitive Linguistics. En: Geeraerts, D. (Ed.). Cognitive Linguistics: Basic readings. Berlin-New York: Mouton de Gruyter.

Hopper, Paul. (1988). Emergent Grammar and the A priori Grammar Postulate. En: Tannen, D. (Ed.). Linguistics in Context: Connective Observation and Understanding. Ablex: Norwood n ${ }^{\circ}$ 5. pp. 117-134.

Hopper, Paul y Thompson, Sandra. (1984). The discourse basis for lexical categories in universal grammar. Language, Vol. 60, no 4. pp. 703-752.

Huffman, Allan. (2001). The linguistic of William Diver and the Columbia 
School. Word, 52-1, pp. 29-68.

Klein-Andreu, Flora. (1983) Grammar in Style: Spanish Adjective Placement. En: Klein- Andreu, F. (Ed). Discourse Perspectives on Syntax. New York: Academic Press. 43-79.

Klima, Edward y Bellugi, Ursula. (1979). The Signs of Language. Cambridge: Harvard University Press.

Lakoff, George. (1987). Women, fire and dangerous things. Chicago: The University of Chicago Press.

Langacker, Ronald. (1987). Foundations of Cognitive Grammar (Vol I). California: Standford University Press.

Langacker, Ronald. (1991). Foundations of Cognitive grammar. Descriptive application (Vol.II). Stanford: Stanford University Press.

Langacker, Ronald. (2008). Cognitive Grammar. A Basic Introduction. Oxford: Oxford University Press.

MacLaughlin, Dawn. (1997). The Structure of determiner phrases: Evidence from American Sign Language. Tesis de doctorado. Boston: Boston University. Martínez, Angelita. (2006). La frase adjetiva. El orden del sustantivo y del adjetivo. En: Company Company, C. (Coord.). Sintaxis histórica de la lengua española. Vol. 2, Tomo 2, Segunda parte: la frase nominal. 1223-1320.

Martínez, Rocío Anabel. (2012). Primeras evidencias de metáforas conceptuales en la Lengua de Señas Argentina (LSA). V Congreso Internacional de Letras. Transformaciones culturales. Debates de la teoría, la critica y la lingüistica. Buenos Aires, Argentina.

Martínez, Rocío Anabel. (2013a). Algunos problemas de la atribución en la Lengua de Señas Argentina. Ponencia presentada en el Seminario de Lingüistica Cognitiva y Funcionalista. Asociación de lingüistas e investigadores de la Lengua de Signos en Catalunya (LingSiC). 15 de julio de 2013. Barcelona, España.

Martínez, Rocío Anabel. (2013b). Primer análisis de morfemas atributivos en sustantivos concretos de la Lengua de Señas Argentina. Ponencia presentada en el V Simposio de la Asociación Argentina de Lingüistica Cognitiva (AALiCo). Universidad del Litoral. 4 y 5 de noviembre de 2013. Ciudad de Santa Fe, Argentina.

Martínez, Rocío Anabel. (2014a). La dimensión en esquemas nominales concretos de la Lengua de Señas Argentina. Ex Libris no 3, año 3, 135-149.

Martínez, Rocío Anabel. (2014b). Lo viejo, lo antiguo y lo anterior: Semántica de tres adjetivos que indican temporalidad en la lengua de señas argentina. Ponencia presentada en el VI Simposio de la Asociación Argentina de Lingüistica Cognitiva (AALiCo). Facultad de Lenguas, Universidad Nacional de Córdoba. 13 y 14 de noviembre de 2014. Ciudad de Córdoba, Argentina.

Martínez, Rocío Anabel. (2015). Algunos aportes del enfoque cognitivo al estudio de clases de palabras en la Lengua de Señas Argentina. Ponencia presentada 
en el II Congreso de la Delegación Argentina de la Asociación de Lingüistica y Filología de América Latina (ALFAL) y VII Jornadas Internacionales de Investigación en Filología y Lingüistica. Facultad de Humanidades y Ciencias de la Educación, Universidad Nacional de La Plata. 21 al 24 de abril de 2015. La Plata, Argentina.

Martínez, Rocío A. y Morón Usandivaras, Mariana. (2013a). Metonimia e iconicidad cognitiva en señas sustantivas concretas de la Lengua de Señas Argentina (LSA). Signo y Seña, 23, 213-237.

Martínez, Rocío A. y Morón Usandivaras, Mariana. (2013b). El doble mapeo en la Lengua de Señas Argentina: Análisis de señas de la comunicación y la cognición. Lengua de Señas e Interpretación, 4, 37-63.

Massone, M. Ignacia (1993). Diccionario Bilingüe Lengua de Señas Argentina- Español-Inglés (2 vol). Buenos Aires: Sopena Argentina.

Massone, M. Ignacia y Machado, Emilia M. (1994). Lengua de Señas Argentina. Análisis y Vocabulario Bilingüe. Buenos Aires: Edicial.

Massone, María I. y Martínez, Rocío A. (2012). Curso de Lengua de Señas Argentina. Mendoza/Berlín: Cultura Sorda.

Massone, M. Ignacia y Martínez, Rocío A. (2013). La metáfora conceptual en el Discurso Político Sordo. En: Pardo, N. G., García, D., Oteíza, T. y Asqueta, M. C. (Comp.). Estudios del discurso en América Latina. Homenaje a Anamaría Harvey. Bogotá: Asociación Latinoamericana de Estudios del Discurso (ALED). 211-237.

Massone, M. Ignacia, Simón, Marina y Druetta, Juan Carlos. (2003). Arquitectura de la escuela de sordos. Buenos Aires: Libros en Red.

Massone, María I., Curiel, Mónica, Buscaglia, Virginia, Famularo, Rosana, Simón, Marina y Carboni, Ignacio. (2000). La Conversación en la Lengua de Señas Argentina. Buenos Aires: Edicial-Libros en Red.

Padden, Carol. (1983 [1980]). The Deaf Community and the Culture of Deaf People. En: Baker, Charlotte y Battison, Robbin. (Eds.). Sign Language and the Deaf Community. Essays in honor of William. C. Stokoe. USA, National Association of the Deaf, 89-103.

Padden, Carol. (1988). Interaction of morphology and syntax in American Sign Language. New York, NY: Garland Publishing.

Real Academia Española. (2009). Nueva Gramática de la Lengua Española. Madrid: Espasa.

Wilbur, Ronnie. B., Evie Malaia \& Robin Shay. (2012). Degree modification and intensification in ASL adjectives. En: Maria Aloni, Vadim Kimmelman, Floris Roelofsen, Galit Sassoon, Katrin Schulz \& Matthijs Westera (Eds.), 18th Amsterdam Colloquium (Lecture Notes in Computer Science, Volume 7218, Logic, Language and Meaning), 92-101. Berlin: Springer. 
Wilcox, Sherman. (2007). Signed Languages. En: Geeraerts, D. y Cuyckens, H. (Eds). The Oxford Handbook of Cognitive Linguistics. Oxford: Oxford University Press. 